\title{
WE-MQS-VoIP Priority: An enhanced LTE Downlink Scheduler for voice services with the integration of VoIP priority mode
}

\author{
Duy-Huy Nguyen*†, Hang Nguyen*, and Éric Renault* \\ *SAMOVAR, Télécom SudParis, CNRS, Université Paris-Saclay \\ 9 rue Charles Fourier - 91011 Evry Cedex, France \\ ${ }^{\dagger}$ Faculty of Information Technology, Hanoi University of Mining and Geology \\ Duc Thang, Bac Tu Liem, Hanoi, Vietnam
}

\begin{abstract}
The Long Term Evolution (LTE) is a high data rates and fully All-IP network. It is developed to support well to multimedia services such as Video, VoIP, Gaming, etc. So that, the real-time services such as VoIP, video, etc. need to be optimized. Nevertheless, the deployment of such live stream services having many challenges. Scheduling and allocating radio resource are very important in LTE network, especially with multimedia services such as VoIP. When voice service transmitted over LTE network, it is affected by many network impairments where there are three main factors including packet loss, delay, and jitter. This study proposes a new scheduler which is based on VoIP priority mode, Wideband (WB) E-model, QoS- and Channel-Aware (called WE-MQS-VoIP Priority scheduler) for voice services in LTE downlink direction. The proposed scheduling scheme is built based on the WB E-model and Maximum Queue Size (MQS). In addition, we integrate the VoIP priority mode into our scheduling scheme. Since the proposed scheduler considers the VoIP priority mode and user perception, thus, it improves significantly the system performance. The results demonstrate that the proposed scheduler not only meets QoS demands of voice calls but also outperforms Modified Largest Weighted Delay First (M-LWDF) in terms of delay, Packet Loss Rate (PLR) for all number of user (NU) and excepting NU equals 30, respectively. For Fairness Index (FI), cell throughput, and Spectral Efficiency (SE), the difference among the packet schedulers is not significant. The performance evaluation is compared in terms of Delay, PLR, Throughput, FI, and SE.
\end{abstract}

Keywords-AMR-WB; Wideband E-model; VoLTE; VoIP priority mode; User perception

\section{INTRODUCTION}

In the digital device market, there are many smart mobile phones such as iPhone, iPad, Android, etc. which is enough powerful to support various types of multimedia communications such as VoIP, Video, Gaming, etc. This means there need be a high data rate network to well support these services. LTE network is proposed by the Third Generation Partnership Project (3GPP) [1]. It has high data rates, low latency, and is fully packet-switched. In downlink direction, LTE uses Orthogonal Frequency Division Multiple Access (OFDMA). This allows supporting inter-symbol interference and selecting fading. Basic components of LTE network consists of a base station (called eNodeB or eNB) and User Equipments (UEs) in addition to a gateway [2]. The eNB station combines with core network through some standard complicated protocols.
A basic scheduler is carried out by mobile network operators in both eNB and UE for both downlink as well as uplink directions. However, according to the 3GPP, there are no firm specifications for scheduling technique in LTE network. One of the most important modules of packet scheduling is Radio Resource Management (RRM) which decides users will be scheduled. The scheduler should care throughput, service policies to subscribes [3].

In LTE network, a full table defined which contains thresholds of delay and packet loss rate for different service classes [1]. In this table, several or all can be performed by service providers. This table divides resource types in the LTE network into two groups, those are Guaranteed Bit Rate (GBR) and Non-GBR. Table I represents the service classes in LTE network where voice service is a Guaranteed Bit Rate (GRB) service which has the second priority just after IP Multimedia Subsystem (IMS) signaling. However, in order to guarantee voice over LTE (VoLTE) quality is an extreme challenge.

TABLE I: Different service classes with QoS demands in LTE network

\begin{tabular}{|c|c|c|c|c|}
\hline $\begin{array}{l}\text { Bearer } \\
\text { Type }\end{array}$ & Priority & $\begin{array}{c}\text { Packet } \\
\text { Delay } \\
(\mathrm{ms})\end{array}$ & $\begin{array}{l}\text { Packet } \\
\text { Loss } \\
\text { Rate }\end{array}$ & Example services \\
\hline \multirow{4}{*}{$\begin{array}{c}\text { Guaranteed } \\
\text { Bit Rate } \\
\text { (GBR) }\end{array}$} & 2 & 100 & $10^{-2}$ & Voice call \\
\hline & 4 & 150 & $10^{-3}$ & Video call \\
\hline & 3 & 50 & $10^{-3}$ & Real-time online gaming \\
\hline & 5 & 300 & $10^{-6}$ & Video streaming \\
\hline \multirow{5}{*}{ Non-GBR } & 1 & 100 & $10^{-3}$ & IMS signaling \\
\hline & 6 & 300 & $10^{-6}$ & $\begin{array}{l}\text { Video, TCP-based services } \\
\text { (e.g. www, e-mail, chat, FTP, etc.) }\end{array}$ \\
\hline & 7 & 100 & $10^{-6}$ & Voice, video, interactive gaming \\
\hline & 8 & \multirow{2}{*}{300} & $10^{-3}$ & \multirow{2}{*}{$\begin{array}{l}\text { Video, TCP-based services } \\
\text { (e.g. www, e-mail, chat, FTP, etc.) }\end{array}$} \\
\hline & 9 & & $10^{-6}$ & \\
\hline
\end{tabular}

In order to meet different QoS requirements for these service classes, some packet schedulers have been proposed. According to [4], the scheduling strategies for LTE downlink are divided into five groups including:

- (1) Channel-unaware strategies;

- (2) Channel-aware/QoS-unaware strategies;

- (3) Channel-aware/QoS-aware strategies;

- (4) Semi-persistent scheduling for supporting VoIP flows; 


\section{- (5) Energy-aware strategies.}

For voice traffics which are very sensitive to delay and PLR, thus, the Channel-aware/QoS-aware strategies are also very essential for them. In addition, VoLTE is really a VoIP service with the QoS guaranteed, and it is transmitted over a heterogeneous LTE network, thus, it need to have a special priority. Therefore, Semi-persistent scheduling is very essential and suitable for VolTE service. Several well-known scheduling algorithms for group 3 as FLS [5], M-LWDF [6], and EXP/PF [7]. In these schedulers, there is only FLS which guarantees bounded delay for real-time flows, the remaining schedulers transmit data of user in a Transmission Time Interval (TTI) by assigning a chosen priority metric. Nevertheless, due to the lack of delay and PLR thresholds, they are not suitable for supporting simultaneously real-time and non real-time traffic [2]. For group 4, authors in [8] proposed a priority mode for VoIP traffic over $3 \mathrm{G}$ LTE. In their scheduler, VoIP priority mode is executed when there is VoIP packet in the queue. This means VoIP packet is scheduled before any other traffic. In [9], authors proposed a new semi-persistent scheduler. Their scheduling scheme is combination of VoIP priority mode with user coupling. This allows using efficiently system capacity. Authors in [10] proposed a new scheduling algorithm which takes into account parameters of VoIP for voice over LTE network. This algorithm is developed based on the VoIP priority mode in [8] which the metric is changed.

In this paper, we present a new downlink scheduling scheme for voice services in LTE network with the integration of VoIP priority mode. Since VoLTE is deployed in an All-IP network, thus, there need to have a special priority for it. This paper is the extension of the WE-MQS scheduler which was proposed in [11] by modifying resource allocation scheduling method. In order to do this, we propose to integrate VoIP priority mode [8] with the essential modifications. We used the WB E-model to predict MOS and use this score as a main factor in the metric. Besides, we see that, the MQS factor has significant effects on the system performance. In the LTESim [12], this factor is fixed equal to 0. This means the MQS is infinite. So that, in the scheduling process, the MQS is not considered. However, in fact, the MQS should be a finite value because if the MQS value is infinite then the delay will increase and the congestion could be increased. Therefore, the MQS needs to be considered as a essential factor in the metric of the scheduling algorithms.

The proposed scheduler selects UEs that based on their priorities which are computed according to the following factors: the maximum MOS, the minimum remaining queue size, the maximum delay, the channel condition. This means for the UE which has the higher MOS, the lower remaining queue size, the higher maximum delay, and the higher channel condition will have the higher priority. We evaluate the performance of the proposed scheduler with the M-LWDF scheduler in a heterogeneous traffic including VoIP, Video, and non real-time service which is called INF-BUF user. The simulation results were implemented in the LTE system simulator (called LTESim) [12] and were compared in terms of Delay, PLR, Cell throughput, and FI for the number of user from 10 to 50.

The remainder of this paper is described as follows: Overview of the system model is described in section II. In section III, we present the proposed scheduling scheme. The simulation results and performance evaluation are analysed in section IV. The conclusion and future work are shown in section $\mathrm{V}$.

\section{THE SYSTEM MODEL}

\section{A. VoLTE traffic flow}

1) Radio protocol stack: The speech frame is encapsulated by network protocols consisting of Real-time Transport Protocol (RTP), User Datagram Protocol (UDP) and Internet Protocol (IP). And then, it will be packetized by other radio protocols including Packet Data Convergence Protocol (PDCP), Radio Control Link (RLC) and Medium Access Control (MAC) at the corresponding layers. At each radio layer, the corresponding header will be added into the speech packet. This leads to data overhead. Therefore, to reduce the data overhead, Robust Header Compression (RoHC) is deployed at the PDCP layer. This allows saving bandwidth as well as enhancing voice transmission in LTE network. RoHC will presses the header size of IP packet from 40 bytes (with IPv4) or 60 bytes (with IPv6) down to 1 to 4 bytes [13]. In addition, at MAC layer, Hybrid Automatic Repeat Request (HARQ) technique is utilized to retransmit in case of FEC (Forward Error Correction) fails error correction. This allows each speech packet to be retransmitted at least from one to three times. The number of retransmissions depends on the error correction or is configured. The implemented model of VoLTE protocol stack used in LTE-Sim [12] is represented on Figure 1.

2) Source codec: VoLTE uses AMR-WB for source codec. It is a voice codec which has been developed by European Telecommunications Standards Institute (ETSI). Details of this codec is described in [14]. AMR-WB codec utilizes a sampling rate of $16 \mathrm{kHz}$, audio bandwidth is in range of 50-7000 Hz. It includes 9 different codec modes of 0-8 which correspond to 9 source bit rates from 6.6 to $23.85 \mathrm{~Kb} / \mathrm{s}$. Each mode generates a compressed speech frame evry $20 \mathrm{~ms}$. The bits in this frame are ordered according to their importance. They are grouped into three classes with reduced importance called Class A, Class $\mathrm{B}$ and Class $\mathrm{C}$. The number of bits in each class depends on codec mode. AMR-WB packet size depends on the bit rate (mode) such as described in Table II.

TABLE II: Packet sizes of AMR-WB modes

\begin{tabular}{|l|c|c|c|c|c|c|c|c|c|}
\hline \multirow{2}{*}{ Parameter } & \multicolumn{10}{|c|}{ Bit rate (kbps) } \\
\cline { 2 - 11 } & $\mathbf{2 3 . 8 5}$ & $\mathbf{2 3 . 0 5}$ & $\mathbf{1 9 . 8 5}$ & $\mathbf{1 8 . 2 5}$ & $\mathbf{1 5 . 8 5}$ & $\mathbf{1 4 . 2 5}$ & $\mathbf{1 2 . 6 5}$ & $\mathbf{8 . 8 5}$ & $\mathbf{6 . 6}$ \\
\hline Payload size (bits) & 477 & 461 & 397 & 365 & 317 & 285 & 253 & 177 & 132 \\
\hline Frame size (bits) & 488 & 472 & 408 & 376 & 328 & 296 & 264 & 192 & 144 \\
\hline RTP header (bits) & 96 & 96 & 96 & 96 & 96 & 96 & 96 & 96 & 96 \\
\hline Packet size (bits) & $\mathbf{5 8 4}$ & $\mathbf{5 6 8}$ & $\mathbf{5 0 4}$ & $\mathbf{4 7 2}$ & $\mathbf{4 2 4}$ & $\mathbf{3 9 2}$ & $\mathbf{3 6 0}$ & $\mathbf{2 8 8}$ & $\mathbf{2 4 0}$ \\
\hline
\end{tabular}

In LTE network, AMR-WB codecs are configured into 3 configurations [15] as follows:

- Configuration A: 6.6, 8.85, and $12.65 \mathrm{~Kb} / \mathrm{s}$ (Mandatory multi-rate configuration);

- Configuration B: 6.6, 8.85, 12.65, and $15.85 \mathrm{~Kb} / \mathrm{s}$;

- Configuration C: $6.6,8.85,12.65$, and $23.85 \mathrm{~Kb} / \mathrm{s}$.

These configurations are used to simplify notation of bit rate between UE and eNB, thus, will simplify implementation 


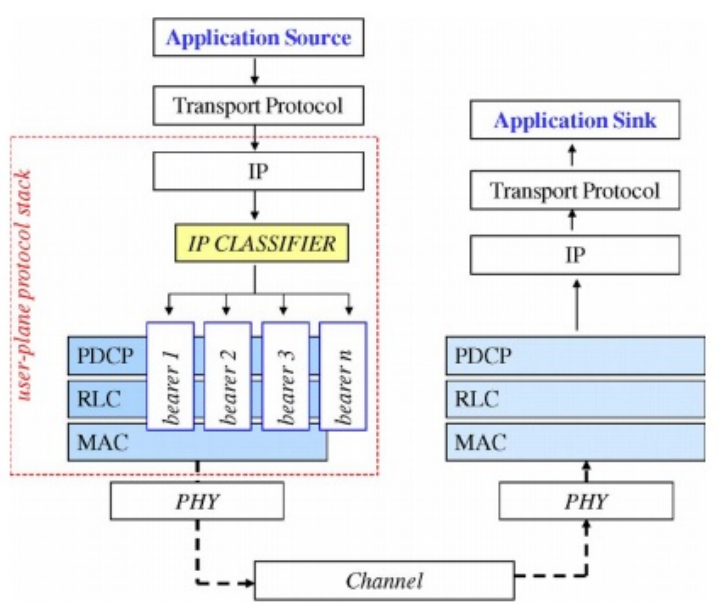

Fig. 1: Implemented model of VoLTE protocol stack [12]

and testing. The remaining bit rates are used for other purposes. In order to choose a bit rate, the receiver measures quality of radio channel. The channel quality indicator (CQI) is used for this purpose. It is defined as an equivalent carrier-tointerference $(\mathrm{C} / \mathrm{I})$ ratio. The $\mathrm{C} / \mathrm{I}$ ratio then compared to a set of predefined thresholds to decide which mode to be used. Switching among modes in a configuration depends on algorithm of rate control. The criterion for mode switching is threshold value of $\mathrm{C} / \mathrm{I}$ ratio. These threshold values depend on the channel condition, frequency hopping scheme, network configuration and other factors. Furthermore, network conditions change over time, so that, even well-selected adaption thresholds will not be best.

3) LTE frame structure: Figure 2 represents the structure of LTE frame for the downlink air interface [16]. In LTE downlink, a frame has length of $10 \mathrm{~ms}$ and is split into 10 subframes in time domain. This means each sub-frame has length of $1 \mathrm{~ms}$ and is split into 2 slots where each slot corresponds to $0.5 \mathrm{~ms}$. In frequency domain, each slot consists of a number of resource blocks (RBs) (from 6 to $10 \mathrm{RBs}$ ). Each slot contains 6 or 7 Orthogonal Frequency Division Multiplexing (OFDM) symbols in normal cyclic prefixes and extended cyclic prefixes, respectively. Each time slot in frequency domain is split into bands of $180 \mathrm{kHz}$ which consists of 12 consecutive subcarriers. Each RB is a basic exchanging information unit in LTE downlink direction. This means RB is a radio resource which is available for user and is defined in both frequency and time domains. In a slot, the number of RBs depends on bandwidth of LTE network [17]. A sub-frame corresponds to a TTI which is minimum transmission unit. Each TTI contains at least one transport block per UE. The size of RB is as same as for all bandwidths [18].

\section{B. Wideband E-model}

WB E-model is a calculative model that is developed and is standardized by ITU-T [19]. The main purpose of this model is to predict quality of wideband audio. The main parameter of this model called R-factor. Its value is in range of 0 129. In order to perform user perception, $\mathrm{R}$-factor is then translated into MOS score. WB E-model is defined such as in the following equation:

$$
R_{w b}=R_{0, w b}-I_{s, w b}-I_{d, w b}-I_{e, e f f, w b}+A
$$

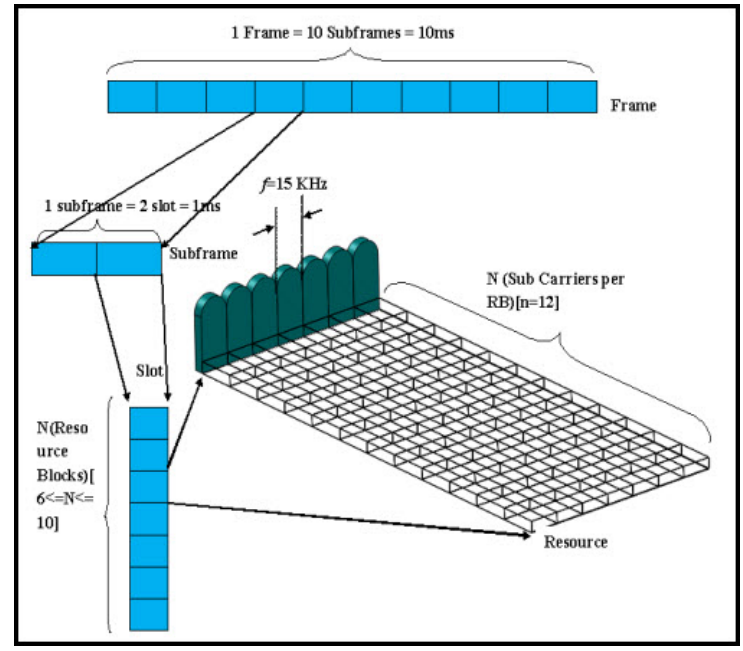

Fig. 2: Resource grid [16]

In which:

- $\quad R_{0, w b}$ : Basic ratio of signal to noise;

- $I_{s, w b}$ : Simultaneous impairment factor which is sum of all impairments that may occur more or less simultaneously with voice transmission. Its default value is 0

- $I_{d, w b}$ : Factor of delay impairment factor, representing all impairments due to delay of voice signals;

- $\quad I_{e, e f f, w b}$ : Factor of equipment impairment, covering the effect of signal distortion due to low bit rates of the codec and packet losses of random distribution;

- A: Advantage factor which represents the fact that some users can accept a degradation of quality due to mobility of mobile networks. Its default value is 0 .

In above parameters, $I_{d, w b}$ and $I_{e, e f f, w b}$ correspond to the effects of end-to-end delay and packet loss while $R_{0, w b}$ and $I_{s, w b}$ don't depend on performance of LTE network. The $R_{w b}$ factor is mapped to MOS score as follows [19]:

$$
\begin{aligned}
& \text { For } R=R_{w b} / 1.29 \\
& \text { MOS }=\left\{\begin{array}{l}
1, \text { if } R<0 \\
1+0.035 \times R+7 \times 10^{-6} \times R \times \\
(R-60) \times(100-R), \text { if } 0 \leq R \leq 100 \\
4.5, \text { otherwise }
\end{array}\right.
\end{aligned}
$$

The relationship among R-factor, user perception, and MOS socre is shown in Table III.

TABLE III: Relationship among R-factor, user perception and MOS score

\begin{tabular}{|c|l|c|}
\hline $\mathbf{R}$ & \multicolumn{1}{|c|}{ User perception } & MOS \\
\hline $90 \leq R<100$ & Very satisfied & $4.3-5.0$ \\
\hline $80 \leq R<90$ & Satisfied & $4.0-4.3$ \\
\hline $70 \leq R<80$ & Some users dissatisfied & $3.6-4.0$ \\
\hline $60 \leq R<70$ & Many users dissatisfied & $3.1-3.6$ \\
\hline $50 \leq R<60$ & Nearly all users dissatisfied & $2.6-3.1$ \\
\hline$R<50$ & Not recommended & $<2.6$ \\
\hline
\end{tabular}

$R_{w b}$ factor is then translated into MOS score accoridng to Equation (2). Next, MOS is translated into user levels of users. 
The value of $R_{0, w b}$ factor for wideband audio in equation (1) equals 129 [20], thus, equation (1) can be rewritten as follows:

$$
R_{w b}=129-I_{d, w b}-I_{e, e f f, w b}
$$

In order to calculate the $R_{w b}$ factor, we must to compute the values of $I_{d, w b}$ and $I_{e, e f f, w b}$ factors. The $I_{d, w b}$ factor is determined as follows [21]:

$$
\begin{aligned}
& I_{d, w b}=0.024 \times D_{e 2 e}+0.11 \times\left(D_{e 2 e}-177.3\right) \times \\
& H\left(D_{e 2 e}-177.3\right)
\end{aligned}
$$

Where $H(x)$ is the Heavyside function:

$$
H(x)=\left\{\begin{array}{l}
0, \text { if } x<0 \\
1, \text { otherwise }
\end{array}\right.
$$

In equation (4), $D_{e 2 e}$ represents end-to-end delay of speech packet. It can be computed via some functions in LTE-Sim. The $I_{e, e f f, w b}$ is determined according to packet loss. According to [20], $I_{e, e f f, w b}$ is counted as the following formula:

$$
I_{e, e f f, w b}=I_{e, w b}+\left(129-I_{e, w b}\right) \times \frac{P_{p l}}{P_{p l}+B_{p l}}
$$

In which:

- $I_{e, w b}$ : Impairment factor without any packet loss;

- $\quad P_{p l}:$ Packet loss rate, is also computed via some functions in LTE-Sim;

- $B_{p l}$ : Codec specific factor that characterizes its robustness against packet loss.

The values of $I_{e, w b}, B_{p l}$ for each mode of AMR-WB codec are represented in Table IV [20].

TABLE IV: Values of $I_{e, w b}$ and $B_{p l}$ for modes of AMR-WB codec

\begin{tabular}{|c|c|c|c|}
\hline $\begin{array}{c}\text { AMR-WB } \\
\text { mode }\end{array}$ & $\begin{array}{c}\text { Bitrate } \\
\text { (bps) }\end{array}$ & $I_{e, w b}$ & $B_{p l}$ \\
\hline 0 & 6.6 & 39 & 12.8 \\
\hline 1 & 8.85 & 25 & 13.5 \\
\hline 2 & 12.65 & 11 & 13 \\
\hline 3 & 14.25 & 10 & 14.1 \\
\hline 4 & 15.85 & 7 & 13.1 \\
\hline 5 & 18.25 & 5 & 12.5 \\
\hline 6 & 19.85 & 4 & 12.3 \\
\hline 7 & 23.05 & 1 & 13 \\
\hline 8 & 23.85 & 6 & 12.2 \\
\hline
\end{tabular}

The $R_{w b}$-factor is then mapped to the MOS via equations (2) . MOS is one of the important factors for the metric in the proposed scheduler.

\section{Semi-persistent scheduling}

This is a hybrid method of Dynamic scheduling and Persistent scheduling [9]. VoIP packets uses a small quantity of control signaling to determine the channel quality after every fixed interval and persistently schedules the VoIP packets. This supports best to VoIP traffics due to its controlled dynamic nature and utilization of small control signaling.

\section{VoIP priority mode}

VoIP priority mode is proposed by Sunggu Choi and others in [8], it allocates RBs for VoIP calls before any other traffic. The limitation of this method is that when VoIP calls density is high, other traffics are not allocated. However, the authors already solved this problem by complementing a duration procedure. It is controlled dynamically to adjust consecutive TTIs according to total drop ratio of the packets measure at the eNodeB. In this mode, RBs are allocated based on Channel Adaptive Fair Queuing [22]. The metric of the scheduler in VoIP priority mode is determined based on queue length and Signal to Interference-plus-Noise Ratio (SINR) and is calculated as the following equation:

$$
w_{i, j}=Q_{l}(i) \times \gamma(i)
$$

Where $\left(Q_{l}\right)$ is queue length and $(\gamma)$ is SINR of active VoIP call $i$. Formula (7) indicates that UE which has the longer queue length and the better channel quality then will have the higher priority. It can be said that, the VoIP priority mode is useful when the density of VoIP calls is high. However, the downside is that other traffic to be starved. Therefore, the duration is deployed. It is controlled dynamically and depends on total of the drop ratio of the packets measured at the eNodeB. A predefined minimum and maximum drop ratio is utilized to adjust VoIP priority duration. Specifically, the maximum count of VoIP priority duration is increased when the drop ratio is less than the minimum threshold, and the maximum count of VoIP priority duration is decreased when the drop ratio exceeds the maximum threshold because in this case there is not enough resources to allocate. If the drop ratio is in range of the min to the max threshold then the duration is kept a constant. For more details of the duration, refer to [8].

\section{THE PROPOSED PACKET SCHEDULER}

In this proposal, we consider the characteristics of VoIP service. This service is sensitive to packet loss and delay, thus, scheduling process should consider various factors. Since VoLTE is transmitted over a fully packet-based network, thus, it needs to be guaranteed QoS to ensure user satisfaction. In this paper, we used the metric which was defined in [11] besides apply the VoIP priority mode for the proposed scheduling scheme.

Firstly, we define a new metric for the proposed scheduling scheme as follows: MOS is a parameter which represents user perception, thus, it should appear in the metric of scheduling algorithms. The higher MOS, the higher user satisfaction. MOS needs be automatically calculated at the receiver and is retransmitted to the eNB via feedback technique. For the MQS, according to our knowledge, there are no articles which mention about it. We think that, this factor has strong effects on the system performance. In the LTE-Sim [12], this factor is fixed equal to 0 . This means the MQS is infinite. Hence the MQS is not considered in the scheduling process. However, in fact, the MQS should be finite. If the MQS value is infinite then the delay will increase and the congestion could be thus increased. Therefore, the MQS should be considered as a necessary factor in the metric of the scheduling algorithms. 
The lack of LTE-Sim software is that it only supports G.729 codec while VoLTE uses AMR-WB codec. G.729 has only an unique mode which has the bitrate of $8 \mathrm{kbps}$ and the packet size of 32 bytes generated in each $20 \mathrm{~ms}$ while AMR-WB has 9 modes. In fact, modes of AMR-WB are changed according to channel condition (i.e. C/I ratio). So, the user perception is calculated at scheduled instant. In the proposed scheduling scheme, we proposed to complement AMR-WB codec into LTE-Sim by reconfiguring some parameters and modifying essential source codes. With the presence of AMR-WB, we can simulate VoLTE traffic more easily.

The primary idea of the proposed scheduler is the consideration of user satisfaction (MOS) and the MQS factor (called also $Q_{i, \max }$ ) included into the metric of the scheduling algorithm. This means the higher MOS and the lower $\left(Q_{i, \max }-Q_{i}\right)$ values, the higher priority for the UE. The fixed maximum time $D_{H O L, i}$ and the maximum probability $\delta_{i}$ are included in the Equations (4) and (6) to calculate the factors of $I_{d, w b}, I_{e, w b}$, respectively. The metric in the proposed scheduling scheme for voice users is defined as follows:

$$
w_{i, j}=\frac{M O S_{i} \times\left(Q_{i, \max }-Q_{i}\right)}{\tau_{i}} \times \frac{r_{i, j}}{\bar{R}_{i}}
$$

Where:

- $Q_{i}, \tau_{i}, r_{i, j}$ and $\bar{R}_{i}$ are similar to ones in the previous formulas;

- $Q_{i, \max }$ : The MQS of the user $i$. This value can be obtained in bytes via some functions in LTE-Sim [12].

For video and non real-time services, we propose to use the metric of the M-LWDF scheduler. The $w_{i, j}$ is a matrix that offers priority for each $R B_{j}$ assigned to $U E_{i}$. It is calculated based on the MOS, the remaining queue size $\left(Q_{i, \max }-Q_{i}\right)$, the maximum time $\tau_{i}$ and the channel condition. MOS is computed at the receiver and is feedbacked to the eNB for making scheduling decision. MOS included in the metric will fully exploit the user perception.

In fact, the AMR-WB mode is dynamically calculated and optimized at the AMR-WB encoder according to channel quality using rate adaptation control algorithm detailed in [15]. The limitation of LTE-Sim is that it supports only G.729 codec for VoIP. Therefore, the proposed scheduler can not get the mode chosen from AMR-WB encoder at Application layer. In order to overtake this issue, we proposed a procedure which allows to choose AMR-WB mode from C/I ration that is available in LTE-Sim. With the proposed procedure, the proposed scheduler can chooses dynamically source codec mode according to channel quality. The threshold values of $\mathrm{C} / \mathrm{I}$ ratio is chosen according to [23] and [24]. The procedures for choosing AMR-WB mode and calculating the metric of the proposed scheduler are detailed in [11]. The procedure of Update AMR-WB packet size is used to update packet size according to channel condition and is used for all schedulers while the procedure of $w_{i j}$ calculation is used only in the proposed scheduler for calculating the metric.

Secondly, we integrate the VoIP priority mode into the proposed scheduling scheme such as represented on Figure 3 . The algorithm priority mode is only enabled when there is VoIP user in the queue. In order to negative effects on other traffics, the duration of VoIP priority mode is deployed. This is detailed in [8].

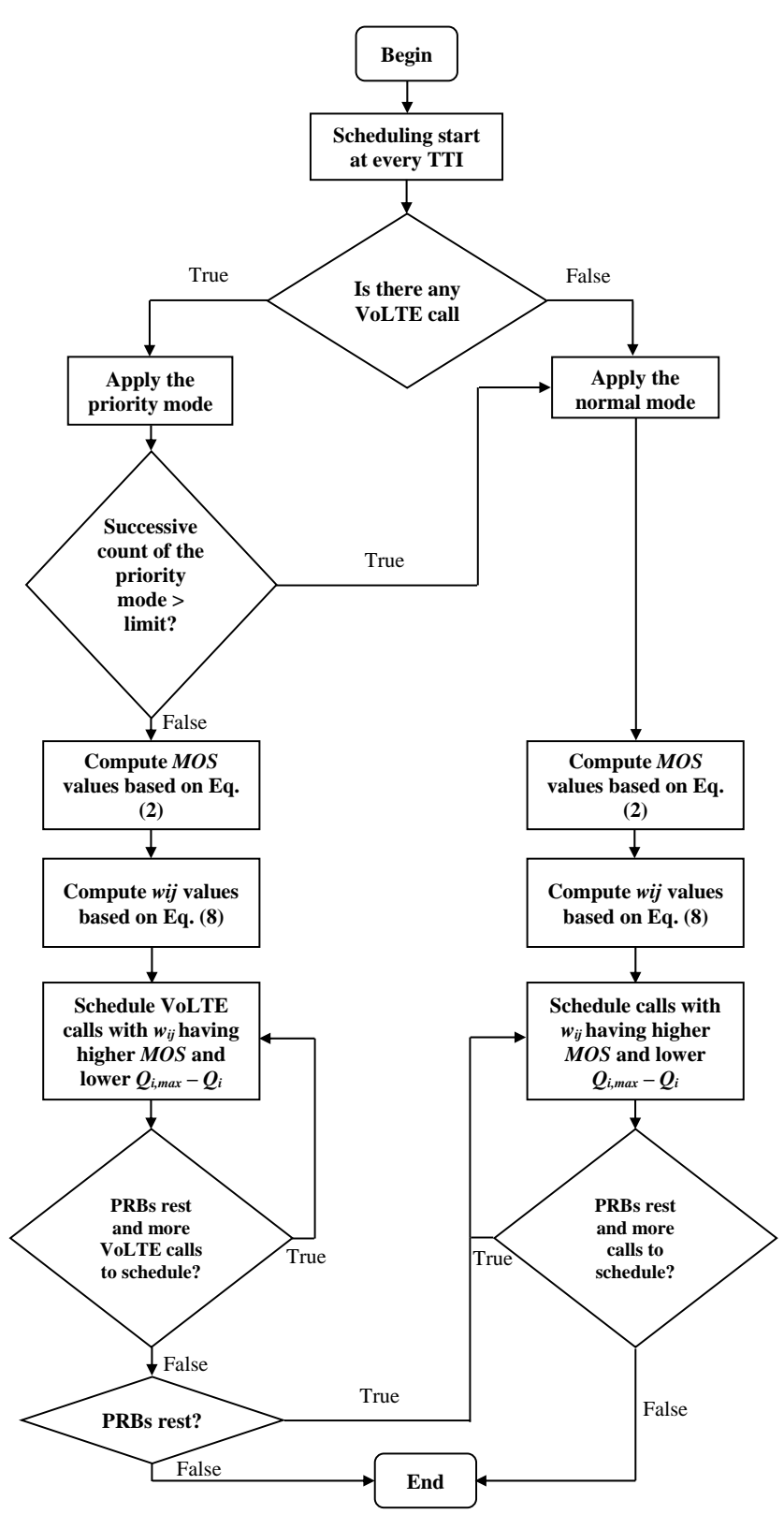

Fig. 3: The proposed scheduling algorithm

\section{Simulation environment and Performance EVALUATION}

\section{A. Simulation environment}

1) Traffic model: In the proposed scenario, the eNodeB lies at center of a macrocell which uses an ommi-directional antenna with a bandwidth of $10 \mathrm{MHz}$. Each UE utilizes a VoIP flow, a Video flow, and a INF-BUF flow. at the same time. For the VoIP flow, a AMR-WB voice stream with bit-rates of 6.6, 8.85, and $12.65 \mathrm{kbps}$ (Configuration A of AMR-WB codec which is mandatory and default for VoLTE service) were used. The voice traffic is a bursty application which is modeled with an ON/OFF Markov chain [25]. For the video traffic, a tracebased application which generates packets using real video trace files with a bit-rate of $242 \mathrm{kbps}$ was used [26] and it is 
also available in [12]. To attain a real simulation of a video streaming, we used an encoded video sequence "foreman.yuv". The encoded spatial resolution CIF $352 \times 288$ with 300 fps has been utilized for the entire simulation.

For the LTE propagation, we use a loss model which is formed by four different models as follows: Path loss, Multipath, Penetration and Shadowing [27].

- Path loss: $P L=128.1+37.6 \times \log (d)$, with $d$ is the distance between the UE and the eNB in $\mathrm{km}$;

- Multipath: Jakes model;

- Penetration loss: $10 \mathrm{~dB}$;

- Shadowing: Log-normal distribution with mean $0 \mathrm{~dB}$ and standard deviation of $8 \mathrm{~dB}$.

2) Simulation parameters: In this study, we investigate and evaluate the performance of M-LWDF and the proposed schedulers in LTE downlink direction. The reason of this choice is that in [11], we proposed the metric for non-VoIP users using the metric of M-LWDF scheduler. The simulation process is performed in a single cell with interference, the number of users is in range of $10 . .50$. UEs move randomly with a speed of $30 \mathrm{~km} / \mathrm{h}$. In the proposed simulation scenario, we consider each user using a VoIP, a Video, and a INFBUF flow. This means the proposed scheduler is evaluated in a heterogeneous LTE network with mobility. For assessing the performance of the system, we use LTE-Sim [12] software. This is a open source framework for researchers and academic community. The basic parameters used in the simulation are represented in the Table V.

TABLE V: Basic parameters for simulating

\begin{tabular}{|l|l|}
\hline \multicolumn{1}{|c|}{ Simulation Parameters } & \multicolumn{1}{c|}{ Values } \\
\hline Simulation duration & $100 \mathrm{~s}$ \\
\hline Frame structure & FDD \\
\hline Cell radius & $1 \mathrm{~km}$ \\
\hline Bandwidth & $10 \mathrm{MHz}$ \\
\hline Video bit-rate & $242 \mathrm{kbps}$ \\
\hline AMR-WB bit-rates & $6.6,8.85,12.65 \mathrm{kbps}$ \\
\hline User speed & $30 \mathrm{~km} / \mathrm{h}$ \\
\hline Number of users & $10,20,30,40,50 \mathrm{UEs}$ \\
\hline Maximum delay & $0.1 \mathrm{~s}$ \\
\hline MQS & $10^{5}$ bytes \\
\hline Maximum VoIP packet drop rate & $5 \%$ \\
\hline Minimum VoIP packet drop rate & $2 \%$ \\
\hline Traffic model & VoIP, Video, and INF-BUF \\
\hline Packet Schedulers & M-LWDF, WE-MQS, and WE-MQS-VoIP Priority \\
\hline
\end{tabular}

\section{B. Performance evaluation}

For assessing the performance of the proposed scheduler, we compare it to the M-LWDF scheduler, and to the scheduler proposed in [11] (called WE-MQS scheduler or normal mode). We assess the performance in terms of delay, PLR, cell throughput, FI and SE. The analysis of the simulation results are represented in the following subsection.

1) Delay: End-to-end delay is the duration required for a packet to be transmitted from source to destination. Figure 4 illustrates the delay of VoIP flow. Such as shown on this figure, the priority mode of the proposed scheduler has the lowest delay when compared to the normal mode and the M-LWDF scheduler. Both modes of the proposed scheduler slightly increase when the NU increase while the M-LWDF heavily increases. In can be said that, when the VoIP priority mode is integrated, the delay decreases significantly.

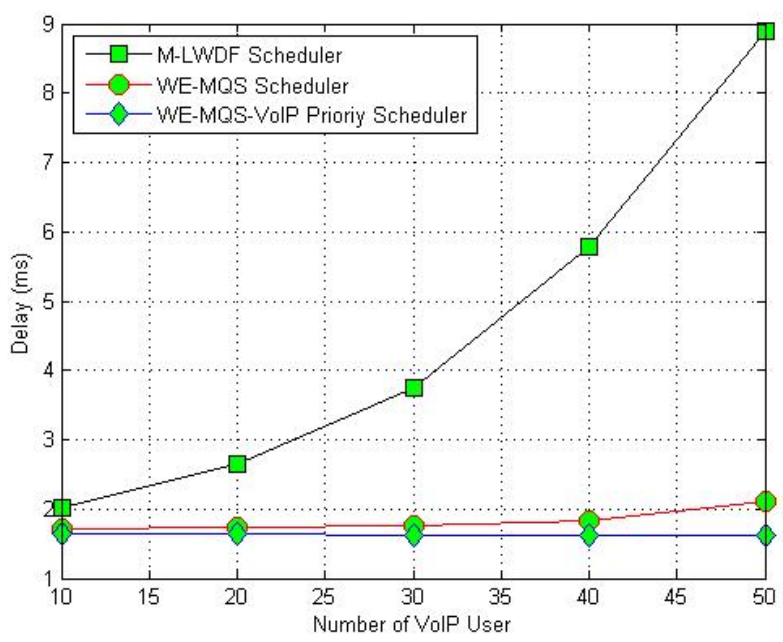

Fig. 4: Delay vs number of VoIP user

2) Packet Loss Rate: PLR represents the failure of one or more packets which are not transmitted successfully to destination. Figure 5 represents the PLR of VoLTE traffic. Normally, the PLR increase when the NU increases. In this study, we assess the system performance in a heterogeneous LTE network with mobility. The results on Figure 5 are quite special, specifically for the normal mode, the PLR decreases when the NU increases. This may be due to the not stable in a real system. In general, the priority mode has the lowest PLR in comparison with two remaining others except when the NU equals 30 .

For the delay and PLR, it can be concluded that the priority mode is very suitable for VoIP service because it has the best performance.

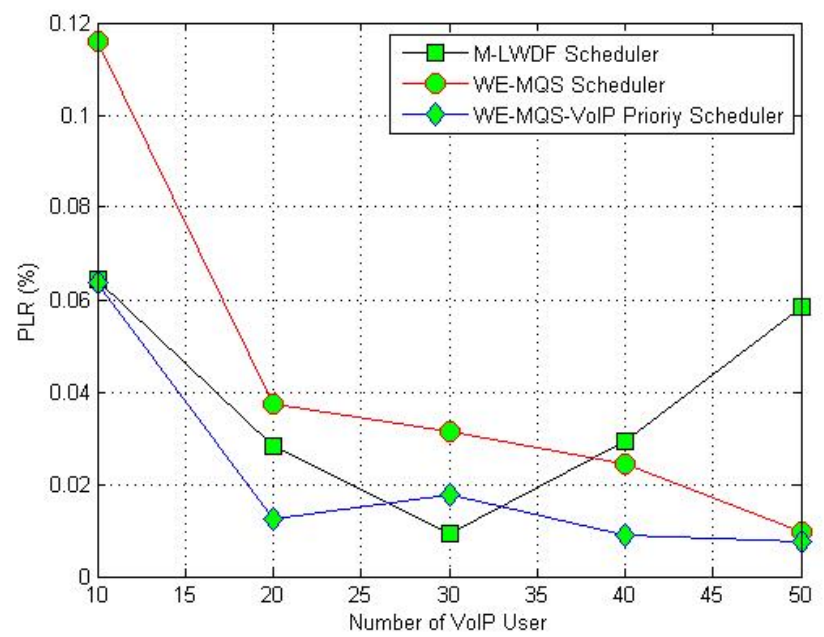

Fig. 5: Packet loss rate vs number of VoIP user

3) Cell throughput: Throughput is a measurement of how many units of information a system can process in a given amount of time. As shown on Figure 6, for the VoIP flow, the cell throughput of all the schedulers increases when the NU 
increases. The priority mode has the best throughput when the $\mathrm{NU}$ in range of 10.30 and slightly reduces when the NU is more than 30, 40 when compared to the M-LWDF and to the normal mode, respectively.

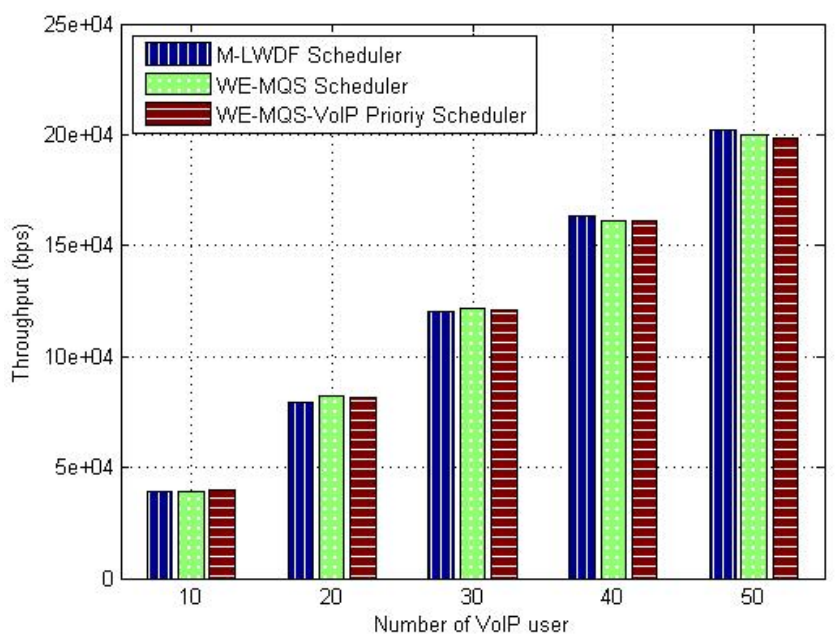

Fig. 6: Throughput vs number of VoIP user

4) Fairness index: FI is a main demand that is used to guarantee a minimum performance to the edge-cell users. For the VoIP flow as shown on Figure 7, the FIs of all schedulers are not stable when the NU increases. Normally, the FI decreases when the NU increases. The VoIP priority mode has the best FI when the NU is less than 30 and has the loewest FI when the NU is more than 30. However, the difference is not significant. The normal mode is always of the middle of two others.

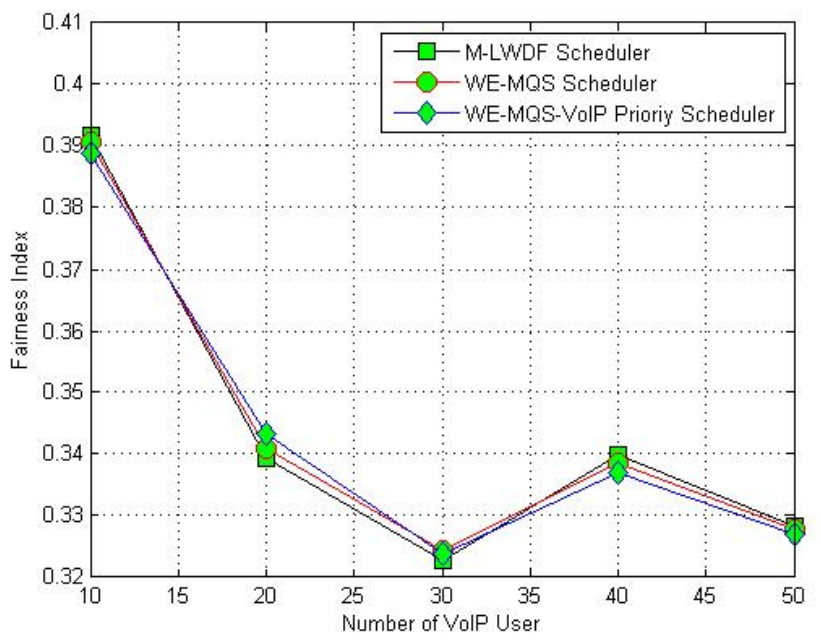

Fig. 7: Fairness Index vs number of VoIP user

5) Spectral efficiency: SE expresses successful usage of radio resources. It is a major principle of the scheduler. SE aims at performance measurements for entire cell. As shown in the Figure 8, the normal mode almost has the lowest SE for all of the NU while the VoIP priority mode has the same SE when compared to the L-MWDF scheduler when the NU is less than 40 . When the NU is more than 40 , the M-LWDF scheduler has the higher SE in comparison with the VoIP priority mode. However, this increase is not very significant.

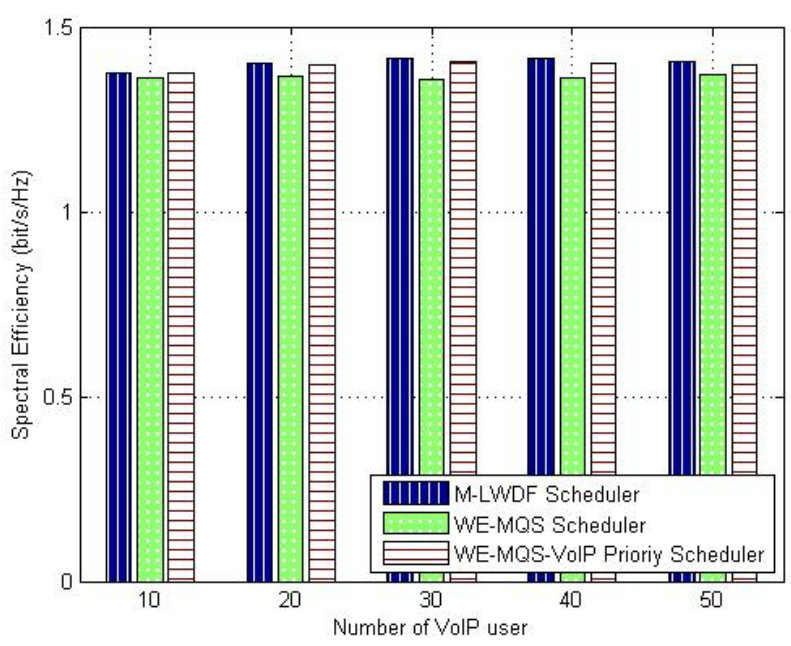

Fig. 8: Spectral efficiency vs number of VoIP user

\section{CONCLUSIONS AND FUtURE WORK}

In this paper, we present a new Channel-, QoS- and QoEAware scheduling scheme with the integration of the VoIP priority mode for voice users in the LTE downlink direction. The main idea in the proposed scheduler is the consideration of user perception (via the MOS score) and the MQS factors into the metric in the proposed scheduler and the presence of VoIP priority mode for VoIP packets. The metric is based on the MOS score, the remaining queue size, the fixed maximum time, and the channel quality. The simulation results show that the proposed scheduler meets QoS requirements for voice services. In addition, it outperforms the M-LWDF scheduler and the normal mode for delay and PLR. With the integration of the VoIP priority mode, the proposed scheduler is enabled, thus, it has the lowest delay and PLR. For the throughput, FI, and SE, in general, it nearly has the same performance when compared to the M-LWDF scheduler. The advantage of the proposed scheduler is that it takes the user satisfaction and the remaining queue size into account and the presence of the VoIP priority mode, thus, it enables the priority for VoIP packets than other traffics. In addition, the proposed scheduler integrates the AMR-WB codec which is mandatory for VoLTE. This overcomes the limitation of the LTE-Sim that it supports only G.729 codec for VoIP application.

It can be said that when considering the MOS and the MQS as factors for the metric in the proposed scheduler and the integration of the VoIP priority mode, the system performance has been improved significantly. Through all simulation results, it can be said that the proposed scheduler has the best performance for VoIP users. Therefore, it is can be said that the proposed scheduler is very suitable and efficient for voice services in the LTE downlink direction. For the future work, we will build a framework to measure voice quality for the proposed scheduler.

\section{REFERENCES}

[1] 3GPP, http://www.3gpp.org. 
[2] S. Ali and M. Zeeshan, "A utility based resource allocation scheme with delay scheduler for lte service-class support," in Wireless Communications and Networking Conference (WCNC), 2012 IEEE. IEEE, 2012, pp. 1450-1455.

[3] M. Alasti, B. Neekzad, J. Hui, and R. Vannithamby, "Quality of service in wimax and lte networks [topics in wireless communications]," Communications Magazine, IEEE, vol. 48, no. 5, pp. 104-111, 2010.

[4] F. Capozzi, G. Piro, L. A. Grieco, G. Boggia, and P. Camarda "Downlink packet scheduling in lte cellular networks: Key design issues and a survey," Communications Surveys \& Tutorials, IEEE, vol. 15, no. 2, pp. 678-700, 2013.

[5] G. Piro, L. A. Grieco, G. Boggia, R. Fortuna, and P. Camarda, "Twolevel downlink scheduling for real-time multimedia services in lte networks," Multimedia, IEEE Transactions on, vol. 13, no. 5, pp. 10521065, 2011.

[6] P. Ameigeiras, J. Wigard, and P. Mogensen, "Performance of the mlwdf scheduling algorithm for streaming services in hsdpa," in Vehicular technology conference, 2004. VTC2004-Fall. 2004 IEEE 60th, vol. 2. IEEE, 2004, pp. 999-1003.

[7] J.-H. Rhee, J. M. Holtzman, and D.-K. Kim, "Scheduling of real/nonreal time services: adaptive exp/pf algorithm," in Vehicular Technology Conference, 2003. VTC 2003-Spring. The 57th IEEE Semiannual, vol. 1. IEEE, 2003, pp. 462-466.

[8] S. Choi, K. Jun, Y. Shin, S. Kang, and B. Choi, "Mac scheduling scheme for voip traffic service in $3 \mathrm{~g}$ lte," in Vehicular Technology Conference, 2007. VTC-2007 Fall. 2007 IEEE 66th. IEEE, 2007, pp. 1441-1445.

[9] S. Saha and R. Quazi, "Priority-coupling-a semi-persistent mac scheduling scheme for voip traffic on $3 \mathrm{~g}$ lte," in Telecommunications, 2009. ConTEL 2009. 10th International Conference on. IEEE, 2009, pp. 325-329.

[10] R. Musabe, H. Larijani, B. Stewart, and T. Boutaleb, "A new scheduling scheme for voice awareness in $3 \mathrm{~g}$ lte," in Broadband and Wireless Computing, Communication and Applications (BWCCA), 2011 International Conference on. IEEE, 2011, pp. 300-307.

[11] H. N. Duy-Huy Nguyen and É. Renault, "We-mqs: A new lte downlink scheduling scheme for voice services based on user perception," International Journal of Computer Applications, vol. 142, no. 10, pp. 28-36, 2016.

[12] G. Piro, L. A. Grieco, G. Boggia, F. Capozzi, and P. Camarda, "Simulating lte cellular systems: an open-source framework," Vehicular Technology, IEEE Transactions on, vol. 60, no. 2, pp. 498-513, 2011.

[13] C. Bormann, C. Burmeister, M. Degermark et al., "Robust header compression (rohc)," RFC 3095, June, Tech. Rep., 2001.

[14] I. Rec, "G. 722.2 (2003) wideband coding of speech at around 16kbit/s using adaptive multi-rate wideband (amr-wb)," International telecommunication union, Geneve, Switzerland, 2003.

[15] 3GPP, "Inband Tandem Free Operation (TFO) of speech codecs; Service description; Stage 3," 3rd Generation Partnership Project (3GPP), TS 28.062, 12 2009. [Online]. Available: http://www.3gpp. org/ftp/Specs/html-info/28062.htm

[16] R. Musabe and H. Larijani, "Cross-layer scheduling and resource allocation for heterogeneous traffic in $3 \mathrm{~g}$ lte," Journal of Computer Networks and Communications, vol. 2014, 2014.

[17] F. Semiconductor, "Long term evolution protocol overview," White Paper, Document No. LTEPTCLOVWWP, Rev 0 Oct, 2008.

[18] 3GPP, "Evolved Universal Terrestrial Radio Access (E-UTRA); Physical channels and modulation," 3rd Generation Partnership Project (3GPP), TS 36.211, 03 2010. [Online]. Available: http: //www.3gpp.org/ftp/Specs/html-info/36211.htm

[19] I. Rec, “G. 107.1, wideband e-model," Int. Telecomm. Union, Geneva, 2011.

[20] S. Möller, A. Raake, N. Kitawaki, A. Takahashi, and M. Wältermann, "Impairment factor framework for wide-band speech codecs," Audio, Speech, and Language Processing, IEEE Transactions on, vol. 14, no. 6, pp. 1969-1976, 2006.

[21] C. Olariu, M. O. Foghlu, P. Perry, and L. Murphy, "Voip quality monitoring in lte femtocells," in Integrated Network Management (IM), 2011 IFIP/IEEE International Symposium on. IEEE, 2011, pp. 501508.

[22] L. Wang, Y.-K. Kwok, W.-C. Lau, and V. K. Lau, "Channel adaptive fair queueing for scheduling integrated voice and data services in multicode cdma systems," Computer Communications, vol. 27, no. 9, pp. 809-820, 2004.

[23] A. Technologies, Available:http://rfmw.em.keysight.com/rfcomms/ refdocs/gsm/default.htm\#gprsla_amr_bse_config.html\#CBDDEBCD, May 2010.

[24] —, Available:http://rfmw.em.keysight.com/rfcomms/refdocs/gsm/ gprsla_wb_amr_bse_config.html, May 2010.

[25] C.-N. Chuah and R. H. Katz, "Characterizing packet audio streams from internet multimedia applications," in Communications, 2002. ICC 2002. IEEE International Conference on, vol. 2. IEEE, 2002, pp. 1199-1203.

[26] M. Reisslein, L. Karam, and P. Seeling, "H. 264/AVC and SVC Video Trace Library: A Quick Reference Guide http://trace. eas. asu. edu," 2009.

[27] 3GPP, "Requirements for Evolved UTRA (E-UTRA) and Evolved UTRAN (E-UTRAN)," 3rd Generation Partnership Project (3GPP), TR 25.913, 12 2009. [Online]. Available: http://www.3gpp.org/ftp/Specs/ html-info/25913.htm 